\title{
The Expected Rate of Gamma\#Ray Burst Afterglows in Supernova Searches
}

\section{Citation}

Woods, Eric, and Abraham Loeb. 1998. "The Expected Rate of Gamma\#Ray Burst Afterglows in Supernova Searches." The Astrophysical Journal 508 (2): 760-66. https://doi.org/10.1086/306437.

\section{Permanent link}

http://nrs.harvard.edu/urn-3:HUL.InstRepos:41417367

\section{Terms of Use}

This article was downloaded from Harvard University's DASH repository, and is made available under the terms and conditions applicable to Other Posted Material, as set forth at http:// nrs.harvard.edu/urn-3:HUL.InstRepos:dash.current.terms-of-use\#LAA

\section{Share Your Story}

The Harvard community has made this article openly available.

Please share how this access benefits you. Submit a story.

\section{Accessibility}




\title{
The Expected Rate of Gamma-Ray Burst Afterglows In Supernova Searches
}

\author{
Eric Woods ${ }^{1}$ and Abraham Loeb ${ }^{2}$
}

Astronomy Department, Harvard University, 60 Garden St., Cambridge, MA 02138

\begin{abstract}
We predict the rate at which Gamma-Ray Burst (GRB) afterglows should be detected in supernova searches as a function of limiting flux. Although GRB afterglows are rarer than supernovae, they are detectable at greater distances because of their higher intrinsic luminosity. Assuming that GRBs trace the cosmic star formation history and that every GRB gives rise to a bright afterglow, we find that the average detection rate of supernovae and afterglows should be comparable at limiting magnitudes brighter than $K=18$. The actual rate of afterglows is expected to be somewhat lower since only a fraction of all $\gamma$-ray selected GRBs were observed to have associated afterglows. However, the rate could also be higher if the initial $\gamma$-ray emission from GRB sources is more beamed than their late afterglow emission. Hence, current and future supernova searches can place strong constraints on the afterglow appearance fraction and the initial beaming angle of GRB sources.
\end{abstract}

Subject headings: gamma rays: bursts

\section{Introduction}

Since their discovery in the late 1960's (Klebasadel et al. 1973) through early 1997, Gamma-Ray Bursts (GRBs) had defied all attempts to determine their distance scale conclusively. The Burst And Transient Source Experiment (BATSE) on board the Compton Gamma-Ray Observatory (GRO) showed that the burst population is highly isotropic (Meegan et al. 1993; Briggs et al. 1993), suggesting that bursts occur at cosmological distances or in an extended Galactic halo. Moreover, the cumulative number counts of faint bursts deviated from that of a uniform distribution of sources in Euclidean space and flattened at faint fluxes, consistent with the expected effect of a cosmological redshift (Fishman \& Meegan 1995, and references therein). Last year, with the advent of the BeppoSAX satellite (Boella et al. 1997), it became possible to localize GRB sources to within an arcminute on a timescale of hours. Such fast, accurate localizations were quickly followed by the detection of delayed X-ray (Costa et al. 1997), optical (van Paradijs et al.

\footnotetext{
${ }^{1}$ email: ewoods@cfa.harvard.edu

${ }^{2}$ email: aloeb@cfa.harvard.edu
} 
1997), and radio (Frail et al. 1997) counterparts to GRB sources. In particular, FeII and MgII absorption lines were detected at a redshift $z=0.835$ in the spectrum of the optical counterpart to GRB970508 (Metzger et al. 1997), demonstrating conclusively that this burst occurred at a cosmological distance with a redshift $z>0.835$. The isotropy of the burst population and the flattening of their number counts, taken in combination with the fact that the first confirmed redshift for an optical counterpart is high, provides strong evidence that GRB sources are located at cosmological distances.

Most plausible GRB models involve either the collapse of a single massive star (e.g. Usov 1992; Woosley 1993; Paczyński 1998), or the coalescence of two compact objects - two neutron stars or a neutron star and a black hole - in a binary system (e.g. Paczyńsky 1986; Eichler et al. 1989; Narayan et al. 1992; Mochkovitch et al. 1993; Rees 1997). Since the lifetime of these progenitors is short compared to the Hubble time at a redshift $z \lesssim 5$, the cosmic GRB rate should simply be proportional to the star formation rate at these redshifts, without any appreciable delay due to the finite progenitor lifetime. The cosmic rate of massive star formation rate has been determined from the $U$ and $B$-band luminosity density in Hubble Deep Field (Madau et al. 1996; Madau 1996; Madau, Pozzetti, \& Dickinson 1997; Madau 1997). The inferred star formation rate $\dot{\rho}_{\mathrm{s}}(z)$ can then be converted to a GRB explosion rate $R_{\mathrm{GRB}}(z)$, based on the requirement that the latter would fit the observed number count distribution of $\gamma$-ray selected GRBs (Wijers et al. 1997).

Cosmological GRBs are at least $10^{4}$ times rarer than Type II supernovae (SNeII) - possibly even $\sim 10^{6}$ times rarer if GRBs occur primarily at high redshifts following the cosmic star formation history (Wijers et al. 1997). However, at peak luminosity, the GRB afterglows are $\sim 10^{3}-10^{4}$ times brighter than SNeII. In Euclidean space, this would imply that GRBs are detected from a volume bigger by a factor $\sim\left(10^{4}\right)^{3 / 2}=10^{6}$, roughly canceling out the factor by which they are rarer than supernovae. Hence we expect that at some relatively bright limiting flux, the rate of afterglow detections should become comparable to that of SN detections. Current and future supernova searches should provide information about the fraction of GRBs which produce detectable afterglows. The statistics of bursts in 1997 for which afterglows could have been identified implies that this fraction is of order tens of percent (e.g., Castro-Tirado 1998). On the other hand, there could also be a population of afterglows without a GRB precursor. This would occur if the source emits a jet from which the $\gamma$-ray emission is more beamed than the subsequent optical afterglow radiation due to the deceleration of the jet by the ambient gas and the corresponding decline in its relativistic beaming with time (Rhoads 1997). A jet geometry would imply a higher rate of afterglow detections in supernova searches.

In this Letter, we predict the detection frequency of GRB afterglows as a function of limiting flux at various observed wavelengths, and compare this rate with the analogous predictions for $\mathrm{SNe}$ Type Ia and Type II at high redshifts. We assume throughout a flat, $\Omega=1, \Lambda=0$, cosmology, with a Hubble constant $H_{0}=50 \mathrm{~km} \mathrm{~s}^{-1} \mathrm{Mpc}^{-1}$. 


\section{Input Parameters}

The inferred energy release in cosmological GRBs and their afterglows is comparable to the binding energy of a neutron star, $\sim 10^{53}$ ergs. We therefore assume that GRBs, like supernova explosions, have evolved stars as their progenitors. Thus, the cosmic GRB and SN rates should both trace the cosmic star formation history. For progenitors with an effective lifetime $\tau$, the corresponding event rate at time $t>\tau$ will be proportional to the star formation rate at time $(t-\tau)$. Following Dwek (1997), we get for the rate of supernovae Type Ia (SNeIa) per comoving volume,

$$
R_{\mathrm{SNIa}}(t)=\frac{\beta \int_{3}^{16} \phi\left(m_{\mathrm{b}}\right) d m_{\mathrm{b}} \int_{\mu_{\mathrm{m}}}^{0.5} \dot{\rho}_{\mathrm{s}}\left[t-\tau\left(\mu m_{\mathrm{b}}\right)\right] f(\mu) d \mu}{\int_{m_{\mathrm{u}}}^{m_{\mathrm{u}}} m \phi(m) d m},
$$

where $\dot{\rho}_{\mathrm{S}}$ is the stellar mass formed per unit comoving volume per unit time, $m$ denotes masses in solar units, $\phi\left(m_{\mathrm{b}}\right)$ is the initial mass function (IMF) for binary systems with total mass in the range $m_{1}<m_{\mathrm{b}}<m_{\mathrm{u}}, f(\mu)=24 \mu^{2}$ is the distribution of secondary-to-total-binary-mass ratios $\mu \equiv m / m_{\mathrm{b}}, \tau(m)$ is the lifetime of a progenitor star of mass $m$, and $\beta<1$ is the SNIa rate amplitude, an adjustable parameter. We find that $\beta=0.05$ yields the best fit to the local and high-redshift supernova observations, and adopt this value. The lower limit in the second integral of the numerator is $\mu_{\mathrm{m}}=\max \left[m(t) / m_{\mathrm{b}},\left(m_{\mathrm{b}}-8\right) / m_{\mathrm{b}}\right]$, where $m(t)$ is the mass of stars which are turning off the main sequence at time $t$, i.e. the inverse of $\tau(m)$ (Greggio \& Renzini 1983). This lower limit take into account the fact that stars more massive than the turnoff mass should not be included in the integral. We assume a Salpeter (1955) IMF, $\phi(m) \propto m^{-2.35}$, with $m_{1}=0.1$ and $m_{\mathrm{u}}=125$. For Type II supernovae, the progenitor lifetime is negligible compared to the Hubble time, and so we take $\tau=0$,

$$
R_{\mathrm{SNII}}(t)=\frac{\dot{\rho}_{\mathrm{S}}(t)\left[\int_{16}^{m_{\mathrm{u}}} \phi(m) d m+(1-\beta) \int_{8}^{16} \phi(m) d m\right]}{\int_{m_{1}}^{m_{\mathrm{u}}} m \phi(m) d m} .
$$

The second term in the numerator accounts for single stars and members of binary systems which do not lead to Type Ia supernovae.

If GRBs have stellar progenitors, then their rate also traces the star formation rate. This scenario was investigated by Wijers et al. (1997), who also assumed that the progenitors are short-lived compared to the Hubble time $(\tau=0)$, and derived a best-fit constant of proportionality between the GRB rate $R_{\mathrm{GRB}}$ and the star formation rate $\dot{\rho}_{\mathrm{s}}$. Given our assumptions about the IMF, their result translates to

$$
R_{\mathrm{GRB}}(t)=7.1 \times 10^{-7} R_{\mathrm{SNII}}(t) .
$$

Note that this rate is substantially lower (by a factor $\sim 150$ ) than the best-fit rate derived by assuming a non-evolving burst population, $R_{\mathrm{GRB}} \sim 10^{-4} R_{\mathrm{SNII}}$. However, Wijers et al. (1997) also obtain a best-fit GRB standard-candle luminosity of $1.6 \times 10^{52} \mathrm{erg} \mathrm{s}^{-1}$ (assuming $\Omega=1$, $H_{0}=50 \mathrm{~km} \mathrm{~s}^{-1} \mathrm{Mpc}^{-1}$ ), which is $\sim 20$ times brighter than that obtained for a non-evolving 
source population. In the following we will consider the best-fit rates for both GRB sources which are evolving (following the cosmic star formation history) or non-evolving.

Next, let us consider a population of transient sources, which are standard candles in peak flux and are characterized by a comoving rate per unit volume $R(z)$. The observed number of new events per unit time brighter than flux $F_{\nu}$ at observed wavelength $\lambda$ for such a population is given by

$$
\dot{N}\left(F_{\nu} ; \lambda\right)=\int_{0}^{z_{\max \left(F_{\nu} ; \lambda\right)}} R(z)(1+z)^{-1}\left(d V_{\mathrm{c}} / d z\right) d z,
$$

where $z_{\max }\left(F_{\nu}, \lambda\right)$ is the maximum redshift at which a source will appear brighter than $F_{\nu}$ at an observed wavelength $\lambda=c / \nu$, and $d V_{\mathrm{c}}$ is the cosmology-dependent comoving volume element. The above integrand includes the $(1+z)$ reduction in the apparent rate due to the cosmic time dilation. For $\Omega=1, \Lambda=0$, the comoving volume element covered by solid angle $d \Omega$ and redshift interval $d z$ is given by

$$
d V_{\mathrm{c}}=4\left(\frac{c}{H_{0}}\right)^{3} \frac{(1+z-\sqrt{1+z})^{2}}{(1+z)^{7 / 2}} d \Omega d z
$$

Equation (4) is appropriate for a threshold experiment, such as BATSE, which monitors the sky continuously and triggers when the detected flux exceeds a certain value, and hence identifies the most distant sources only when they are near their peak flux. For search strategies which involve taking a series of "snapshots" of a field and looking for variations in the flux of sources in successive images, one does not necessarily detect most sources near their peak flux. In this case, the total number of events (not per unit time) brighter than $F_{\nu}$ at observed wavelength $\lambda$ is given by

$$
N\left(F_{\nu} ; \lambda\right)=\int_{0}^{\infty} R(z) t_{\star}\left(z ; F_{\nu}, \lambda\right)\left(d V_{\mathrm{c}} / d z\right) d z,
$$

where $t_{\star}\left(z ; F_{\nu}, \lambda\right)$ is the rest-frame duration over which an event will be brighter than the limiting flux $F_{\nu}$ at redshift $z$. This is a naive estimate of the so-called "control time"; in practice, the effective duration over which an event can be observed is shorter, owing to the image subtraction technique, host galaxy magnitudes, and a number of other effects which reduce the detection efficiency (Pain et al. 1996).

In applying equations (1)-(6), we use the star formation rate $\dot{\rho}_{\mathrm{s}}(z)$ determined by Madau (1997) and convert cosmic time to redshift according to the standard cosmological formulae. For the ages of the SNIa progenitors we use the calculations by Schaller et al. (1992). To determine the maximum redshift $z_{\max }$ and the effective duration $t_{\star}$ for both SNe and GRBs, we use the solar-metallicity, time-dependent, spectral models of Eastman et al. (1994) for SNeII, the template lightcurves of Riess, Press, \& Kirshner (1996) together with the spectral models of Nugent et al. (1997) for SNeIa - extended out to $2.2 \mu \mathrm{m}$ with a simple blackbody fit, and the broken power law model of Waxman (1997) for GRB afterglows. In all cases, we truncate the emission spectrum at rest wavelengths shorter than $\sim 10^{3} \AA$, to reflect the absorption by HI beyond the Lyman-limit in the local environment of the source and the intergalactic medium. For the afterglows, we modify Waxman's choice of parameters after peak luminosity so as to match the faster decay slope 
observed for GRB970228 (Galama et al. 1997; Fruchter et al. 1998) and GRB970508 (Galama et al. 1998), namely $L_{\nu} \propto t^{-1.1}$. We also introduce a cutoff time $t_{\text {cut }}$ beyond which we truncate the afterglow flux so that the source counts will converge (see $\S 3$ for the effect of varying $t_{\text {cut }}$ ). The resulting GRB spectrum as a function of time is

$$
L_{\nu}(t)=2.94 \times 10^{30} \eta\left(\frac{t_{\text {day }}}{t_{\lambda}}\right)^{\alpha} \operatorname{erg~s}^{-1} \mathrm{~Hz}^{-1},
$$

where $t_{\text {day }} \equiv(t /$ day $), \alpha=0.5$ for $t_{\text {day }} \leq t_{\lambda}, \alpha=-1.1$ for $t_{\lambda}<t_{\text {day }}<t_{\text {cut }}$, and $t_{\lambda} \equiv\left(1.41 \times 10^{14} \mathrm{~Hz} / \nu\right)^{2 / 3}$ days $=(\lambda / 2.13 \mu \mathrm{m})^{2 / 3}$ days. The parameter $\eta$ reflects the choice of a typical GRB redshift, with $\eta=1$ for a non-evolving burst population - corresponding to a typical redshift $z \sim 1$. On the other hand, Wijers et al. (1997) derive a characteristic $\gamma-$ ray luminosity which is $\sim 20$ times higher than that obtained for a non-evolving population. The afterglow luminosity depends on the total fireball energy (Waxman 1997), which is proportional to the product of the $\gamma$-ray luminosity and the rest-frame duration. Taking all of these dependences into account, we derive $\eta \sim 10$ for a population which traces the star formation history. We express the afterglow emission in terms of the luminosity per unit frequency, since detector sensitivities are often expressed in Janskys ( $1 \mathrm{Jy}=10^{-23} \mathrm{erg} \mathrm{s}^{-1} \mathrm{~cm}^{-2} \mathrm{~Hz}^{-1}$ ). From equation (7), we have for GRB afterglows

$$
\begin{aligned}
t_{\star}\left(z ; F_{\nu}, \lambda\right) & =\min \left\{t_{\text {cut }}, A\left(z ; F_{\nu}\right)^{-0.9} t_{\lambda /(1+z)}\right\}-A\left(z ; F_{\nu}\right)^{2} t_{\lambda /(1+z)} \\
A\left(z ; F_{\nu}\right) & \equiv \frac{4.0 \times 10^{2} \eta^{-1}}{(1+z)}\left[\frac{F_{\nu}}{\mathrm{Jy}}\right]\left[\frac{D_{\mathrm{L}}(z)}{\mathrm{Gpc}}\right]^{2}
\end{aligned}
$$

where $D_{\mathrm{L}}(z)$ is the cosmology-dependent luminosity distance, $D_{\mathrm{L}}(z)=\left(2 c / H_{0}\right)(1+z-\sqrt{1+z})$ for $\Omega=1$ and $\Lambda=0$.

\section{Results}

We apply equation (4) to determine the average rate at which GRB afterglows, SNeIa, and SNeII should be detected in a field of $1 \mathrm{deg}^{2}$ as a function of limiting flux. Figure 1 shows the results for different spectral bands, namely the $K\left(\lambda_{\text {eff }}=2.2 \mu \mathrm{m}\right), R\left(\lambda_{\text {eff }}=7000 \AA\right), B$ (effective wavelength, $\left.\lambda_{\text {eff }}=4400 \AA\right)$, and $U\left(\lambda_{\text {eff }}=3650 \AA\right)$ bands. The $B$-band rate of $\sim 4800$ SNeII $\mathrm{yr}^{-1} \mathrm{deg}^{-2}$ brighter than $1 \mathrm{nJy}$ is slightly lower than a recent prediction (Madau 1998) of 25-35 SNeII $\mathrm{yr}^{-1}$ per $4^{\prime} \times 4^{\prime}$ field of view of the Next Generation Space Telescope (NGST, Mather \& Stockman 1996), where the range of values corresponds to a range of assumptions about the amount of dust extinction between the observer and the sources. Our slight underestimate of $\sim 21 \mathrm{SNeII} \mathrm{yr}^{-1}$ per NGST field results from the fact that we do not correct the star formation rate for the effects of dust extinction. Our predictions from equations (1)-(2) for the local $(z=0)$ SNIa and SNII rates are consistent with recent observations (Cappellaro et al. 1997; Sadat et al. 
1998). The SNIa rate we obtain at a redshift $z \sim 0.4$ agrees to within statistical uncertainties with the rate determined from observations of high-redshift SNeIa (Pain et al. 1996). The Type Ia supernova counts overtake the Type II counts at relatively bright fluxes, since SNeIa are an order of magnitude brighter than SNeII and hence dominate the counts in a shallow magnitude-limited sample, even though their absolute rate is a fraction $(\sim 30-50 \%)$ of the Type II rate. For both types of supernovae, we truncate the spectrum at rest wavelengths shorter than $\sim 10^{3} \AA$ due to the expected galactic and intergalactic absorption by neutral hydrogen.

The predicted GRB afterglow rate in Figure 1 is nearly independent of the observed band, since the peak GRB flux in equation (7) is the same at all wavelengths. The sharp "knee" at $10^{-4}-10^{-3} \mathrm{Jy}$ reflects the edge in the spatial distribution of luminous bursts due to the non-Euclidean geometry of the Universe and the sharp decline in the star formation rate at $z>4$. We extrapolate the star formation rate using a power-law dependence on $(1+z)$ at $z>4$. Our extrapolation is consistent with the upper limit on $\dot{\rho}_{\mathrm{s}}(z)$ around $z=5.5$, based on the lack of " $V$ dropout" galaxies in the Hubble Deep Field (Madau 1996). Production of bright afterglows, of the type observed in GRB970228 or GRB970508, requires an ambient gas density of $\sim 1 \mathrm{~cm}^{-3}$ (Waxman 1997), which is typical of the interstellar medium of disk galaxies. Hence, we expect significant Lyman-limit absorption due to $\mathrm{HI}$ in the local burst environment, and so we truncate the afterglow spectrum at rest wavelengths $<10^{3} \AA$. This explains the variation with observed wavelength of the rate of faint afterglows. Note that at all observed wavelengths, the afterglow rate becomes comparable to the Type II SN rate at magnitudes $\sim 18-20$. This means that if afterglows occur with the same frequency as the $\gamma$-ray selected GRBs, then they should turn up with comparable numbers to supernovae in searches of this depth.

Figure 2 shows the results of applying equation (6) to obtain the number of events that are detectable in a single "snapshot", taking into account the effective length of time during which each type of event should be visible at a given sensitivity. Our prediction for the $R$-band counts of SNeIa is consistent with the results of the high-redshift survey of Pain et al. (1996), who found three Type Ia supernovae in the range $21.3<R<22.3$ across a $1.73 \mathrm{deg}^{2}$ area on the sky. Our predicted rate is somewhat higher, due to the fact that our naive estimate of the "control time" $t_{\star}$ assumes $100 \%$ efficiency over the duration of the event, while the actual efficiency (and the corresponding value of $t_{\star}$ ) are lower in a real search. Afterglows are less abundant relative to supernovae in Figure 2 as compared to Figure 1, because the timescale for the decline of their lightcurve around peak flux is an order of magnitude shorter [cf. Eqs. (4) and (6)].

The lower and upper solid lines in Figures 1 and 2 show the predicted afterglow rates with and without evolution. The two lines differ substantially at faint fluxes and coincide to within a factor of a few at bright fluxes. The coincidence at the bright end results from the fact that the adopted GRB luminosities and rates were chosen so as to reproduce the same flux distribution of BATSE-selected GRBs. However, at faint fluxes which require sensitivities greater than that achieved by the BATSE experiment, the counts converge to different values. The no-evolution model predicts many more bursts at redshifts low enough such that the observed frequency falls 
below the Lyman limit at emission.

In order to apply equation (6) to the afterglow population, we needed to assume that the afterglow emission truncates after some time $t_{\text {cut }}$, since otherwise the power-law lightcurve implied by equation (7) would lead to an untruncated power-law in the number counts, and a divergence of the number of afterglows at faint fluxes. Observations indicate that GRB afterglows continue their power-law decline, $L_{\nu} \propto t^{-1.1}$, over a considerable fraction of a year (Galama et al. 1997), and so in Figure 2 we assumed a value of $t_{\text {cut }}=1$ year. The effect of varying this choice of $t_{\text {cut }}$ is illustrated in Figure 3; the position of the number count "knee" shifts to fainter fluxes when $t_{\text {cut }}$ is increased since a larger $t_{\text {cut }}$ corresponds to a larger effective volume being surveyed. The uncertainty concerning $t_{\text {cut }}$ translates to an uncertainty in the expected faint counts. By contrast, the afterglow rate predictions displayed in Figure 1 are effectively independent of the detailed shape of the lightcurve.

\section{Conclusions}

We have predicted the rate at which GRB afterglows should be detected in supernova searches as a function of limiting flux. Requiring that the GRB population would evolve according the cosmic star formation history reduces the number counts of afterglows at faint fluxes by $\sim 2$ orders of magnitude relative to the no-evolution case (see the solid lines in Figs. 1 and 2). Our main result is that the average detection rate of new afterglows (in both evolutionary cases) is comparable to that of supernovae for a continuous search with a magnitude limit of $K=18$ (cf. Fig. 1), assuming that every GRB produces a bright afterglow of the type observed for GRB970228 or GRB970508. However, the number of detectable afterglows in a "snapshot" search (cf. Fig. 2) could be an order of magnitude smaller than the number of detectable supernovae, since the afterglow emission declines around its peak flux an order of magnitude faster. The actual number of afterglows in real surveys might be even lower since only a fraction of all $\gamma$-ray selected GRBs detected in 1997 were observed to give rise to bright afterglows. (One theoretical interpretation of this result could be in terms of the proportionality between the predicted afterglow luminosity and the square root of the ambient gas density (e.g., Waxman 1997). The distribution of afterglow luminosities is expected to reflect the range of gas densities in GRB environments and might span several orders of magnitude.) The reduction factor due to the small appearance probability of bright afterglows might be counteracted by a possible enhancement factor due to beaming. If the initial gamma-ray emission is beamed to an angle $\sim 1 / \gamma_{0}$ where $\gamma_{0}$ is the initial Lorentz factor, while the afterglow radiation is produced when the fireball has been decelerated to a modest Lorentz factor $\gamma_{\mathrm{a}}$, then the number of optically-selected afterglows could be greater by a factor $\sim\left(\gamma_{0} / \gamma_{\mathrm{a}}\right)^{2}$ than the number of $\gamma$-ray selected GRBs (Rhoads 1997). Since typical bursts have $\gamma_{0} \gtrsim 10^{2-3}$ (e.g., Fenimore, Epstein, \& Ho 1993; Woods \& Loeb 1995) while the optical afterglow emission occurs at $\gamma_{\mathrm{a}} \sim 10^{1-2}$, this could boost the expected afterglow rates by up to four orders of magnitude. Existing supernova searches (e.g., Garnavich et al. 1998; Perlmutter et al. 1998) 
reach an effective magnitude limit $R \gtrsim 22$ (Kirshner 1998), and could already place meaningful constraints on the beaming of the $\gamma$-ray emission. Future searches might also constrain the appearance probability of the afterglow emission and the evolution of the GRB population.

The Next Generation Space Telescope, with its detection sensitivity of $\sim 10^{-9}$ Jy at $1-3.5 \mu \mathrm{m}$ (Mather \& Stockman 1996), will be able to see GRB afterglows out to redshifts $z \gtrsim 10$, if they exist $^{3}$. Afterglows might be the brightest sources at these redshifts aside from early quasars (Haiman \& Loeb 1997b). Based on the expected supernova rate at $z \gtrsim 5$ (Miralda-Escudé \& Rees 1997), there might be one detectable afterglow per year per $\sim \operatorname{deg}^{2}$ in the no-evolution model. The stretching of the observed lifetime of afterglows from high redshifts and the potential enhancement factor for the afterglow rate due to beaming, might bring the detection of afterglows closer to the realm of feasibility. The discovery of high redshift afterglows could then be used to trace the star formation history out to the "dark ages" of the Universe when the first stars and quasars formed (e.g., Rees 1996; Haiman \& Loeb 1997a,b; Loeb 1997). The optical afterglow radiation could also be used as a means of identifying the reionization redshift through the spectral location of the Gunn-Peterson absorption trough which is produced by the neutral intergalactic medium prior to reionization.

We thank Bob Kirshner for useful discussions and Ron Eastman for providing us with the latest model spectra for Type II supernovae.

\section{REFERENCES}

Boella, G., et al. 1997, A\&AS, 122, 299

Briggs, M. S., et al. 1993, in Proc. of the Huntsville Gamma-Ray Burst Workshop, ed. G. Fishman, J. Brainerd, \& K. Hurley (New York: AIP), 44

Cappellaro, E., Turatto, M., Tsvetkov, D. Yu., Bartunov, O. S., Pollas, C., Evans, R., \& Hamuy, M. 1997, A\&A, 322, 431

Castro-Tirado, A. J. 1998, astro-ph/9803007

Costa, E., et al. 1997, Nature, 387, 783

Dwek, E. 1997, ApJ, submitted, astro-ph/9707024

Eastman, R. G., Woosley, S. E., Weaver, T. A., \& Pinto, P. A. 1994, ApJ, 430, 300

Eichler, D., Livio, M., Piran, T., \& Schramm, D. N. 1989, Nature, 340, 126

\footnotetext{
${ }^{3}$ If GRBs result from binary coalescence events of compact stars, then their abundance might be suppressed as long as the age of the Universe is shorter than the characteristic coalescence time. On the other hand, the initial mass function of the first stars is likely to be tilted towards high mass stars, which are probable progenitors of neutron stars or black holes. This would enhance the abundance of such binaries per generation of stars.
} 
Fenimore, E. E., Epstein, R. I., \& Ho, C. 1993, A\&AS, 97, 59

Ferrini, F., Matteucci, F., Pardi, C., \& Penco, U. 1992, ApJ, 387, 138

Fishman, G. J., \& Meegan, C. A. 1995, ARA \& A, 33, 415

Frail, D. A., et al. 1997, ApJ, 483, L91

Fruchter, A. S., et al. 1998, to appear in Proc. of the 4th Huntsville GRB Symposium, 1997, Eds. C. A. Meegan, R. Preece, \& T. Koshut, astro-ph/9801169

Galama, T. J., et al. 1997, astro-ph/9712322

Galama, T. J., et al. 1998, ApJL, in press, astro-ph/9802160

Garnavich, P. M., et al. 1998, ApJ, 493, L53

Greggio, L., \& Renzini, A. 1983, A\&A, 118, 217

Haiman \& Loeb 1997a, ApJ, 483, 21 . 1997b, ApJ, in press, astro-ph/9710208

Kirshner, R. 1998, private communication

Klebasadel, R. W., Strong, I. B., \& Olson, R. A. 1973, ApJ, 182, L85

Loeb A. 1997, "The First Stars and Quasars In the Universe", in "Science With NGST", Eds. E. Smith \& A. Koratkar, ASP Conf. Series, 133, 73; astro-ph/9704290

Madau, P. 1996, in "Star Formation Near and Far", AIP Conf. Proc. (New York: AIP), astro-ph/9612157

Madau, P. 1997, to appear in "The Hubble Deep Field", ed. M. Livio, S. M. Fall, \& P. Madau, STScI Symposium Series, astro-ph/9709147

Madau, P. 1998, to appear in "The Young Universe: Galaxy Formation and Evolution at Intermediate and High Redshift", ed. S. D’Odorico, A. Fontana, \& E. Giallongo, PASP, astro-ph/9801005

Madau, P., Ferguson, H. C., Dickinson, M. E., Giavalisco, M., Steidel, C. C., \& Fruchter, A. 1996, MNRAS, 283, 1388

Madau, P., Pozzetti, L., \& Dickinson, M. 1997, ApJ, submitted, astro-ph/9708220

Mather, J., \& Stockman, P. 1996, STScI newsletter, 13(2), 15

Meegan, C. A., et al. 1993, in Proc. of the Huntsville Gamma-Ray Burst Workshop, ed. G. Fishman, J. Brainerd, \& K. Hurley (New York: AIP), 3

Metzger, M. R., et al. 1997, Nature, 387, 878

Miralda-Escudé, J., \& Rees, M. J. 1997, ApJ, 478, L57

Mochkovitch, R., Hernanz, M., Isern, J., \& Martin, X. 1993, Nature, 361, 236

Narayan, R., Paczyński, B., \& Piran, T. 1992, ApJ, 395, L83 
Nugent, P., Baron, E., Branch, D., Fisher, A., \& Hauschildt, P. H. 1997, ApJ, 485, 812

Paczyński, B. 1986, ApJ, 308, L43

Paczyński, B. 1998, ApJ, 494, L45

Pain, R., et al. 1996, ApJ, 473, 356

Perlmutter, S., et al. 1998, Nature, 391, 51

Rhoads, J. E. 1997, ApJ, 487, L1

Rees, M. J. 1996, astro-ph/9608196

Rees, M. J. 1997, to appear in Proc. of the Texas symposium, Chicago, Dec. 1996, astro-ph/9701162

Riess, A. G., Press, W. H., \& Kirshner, R. P. 1996, ApJ, 473, 88

Ruiz-Lapuente, P., \& Canal, R. 1998, ApJL, in press, astro-ph/9801141

Sadat, R., Blanchard, A., Guiderdoni, B., \& Silk, J. 1998, A\&A, 331, L96

Salpeter, E. E. 1955, ApJ, 121, 161

Schaller, G., Schaerer, D., Meynet, G., \& Maeder, A. 1992, A\&AS, 96, 269

Usov, V. V. 1992, Nature, 357, 472

van Paradijs, J., et al. 1997, Nature, 386, 686

Waxman, E. 1997, ApJ, 489, L33

Wijers, R. A. M. J., Bloom, J. S., Bagla, J. S., \& Natarajan, P. 1997, MNRAS, submitted, astro-ph/9708183

Woods, E., \& Loeb, A. 1995, ApJ, 453, 583

Woosley, S. E. 1993, ApJ, 405, 273

Yungelson, L., \& Livio, M. 1998, ApJ, in press, astro-ph/9711201 

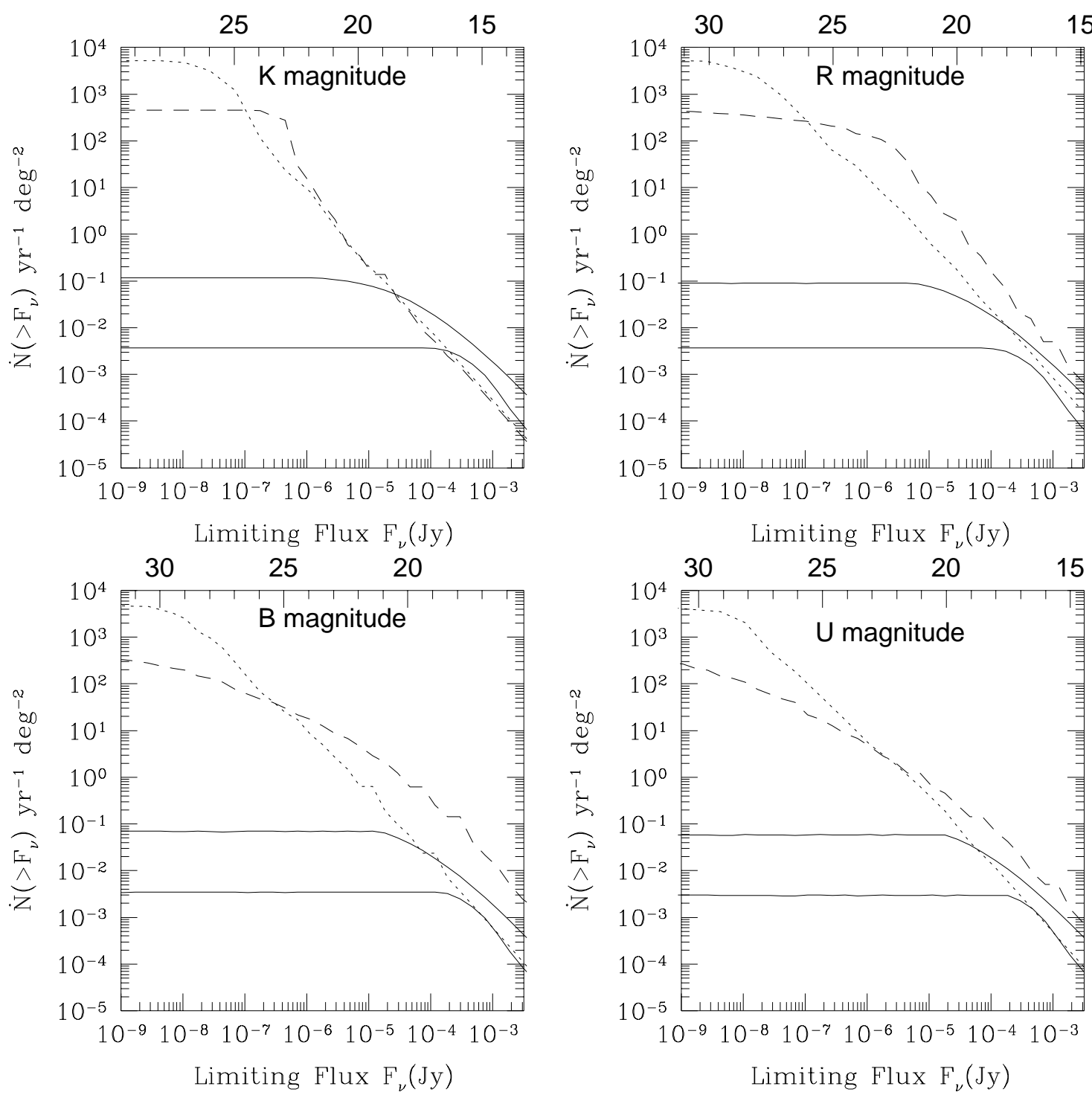

Fig. 1.- Cumulative observed rate $\dot{N}\left(>F_{\nu}\right.$ ) per year per square degree of GRB afterglows (solid curves) at four wavelengths, corresponding to the $K, R, B$, and $U$ bands. The lower solid curve assumes the best-fit rate and luminosity for GRB sources which trace the star formation history (Wijers et al. 1997), while the upper solid curve assumes the best-fit values for a non-evolving GRB population. For comparison, we show the counts for SNe Ia (dashed curves) and SNe II (dotted curves). 

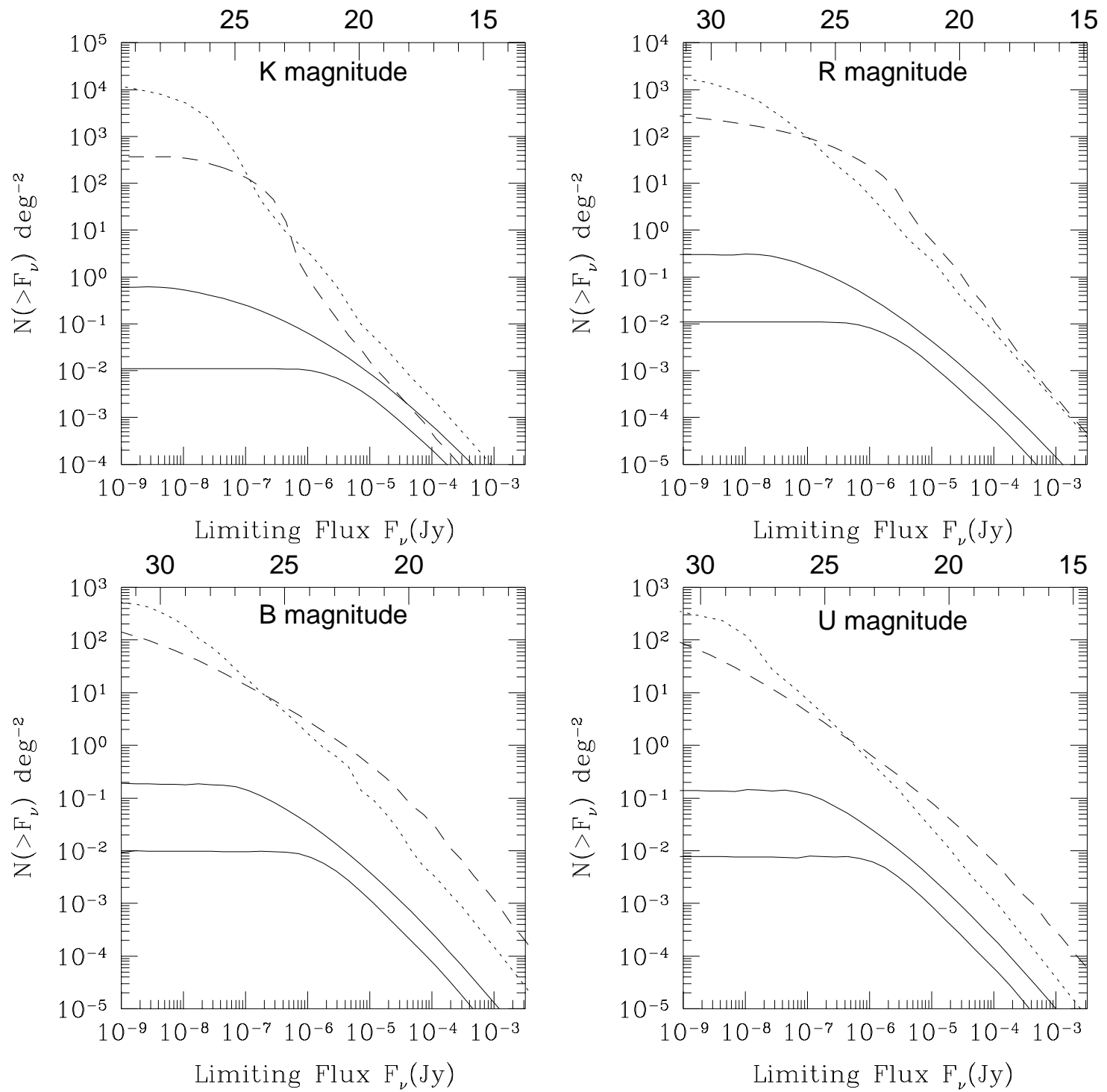

Fig. 2.- Cumulative number counts $N\left(>F_{\nu}\right)$ per square degree for GRB afterglows, with a cutoff time of $t_{\text {cut }}=1$ yr. Notations are the same as in Figure 1. 


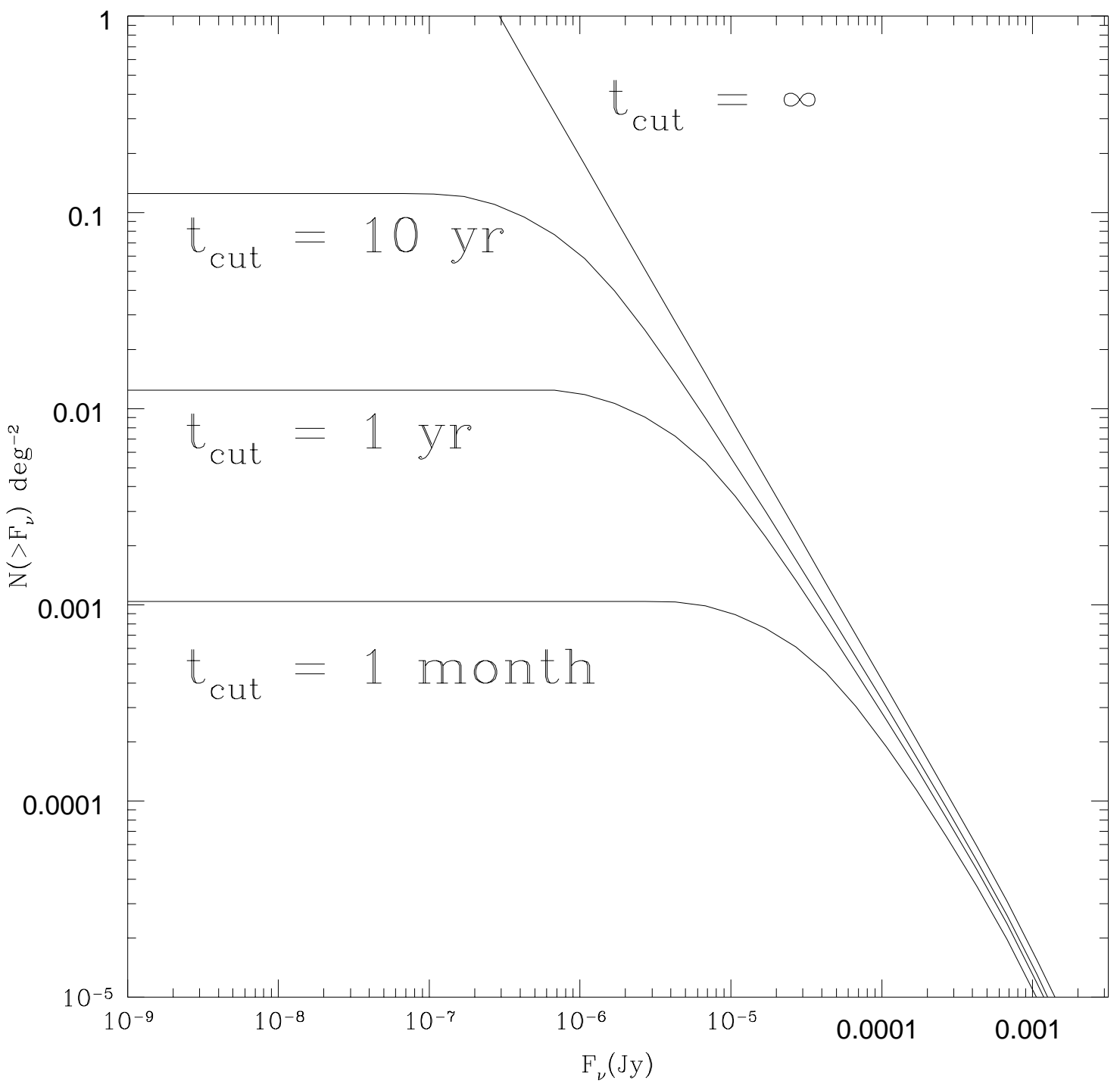

Fig. 3.- Cumulative $B$-band counts of GRB afterglows for various values of the cutoff time $t_{\text {cut }}$. From top to bottom, the curves are for $t_{\text {cut }}=\infty, 10$ years, 1 year, and 1 month. 